'P Heinz, ${ }^{2}$ S Rann, 'R Ross Russell. 'Department of Paediatrics, Addenbrooke's Hospital; ${ }^{2}$ Shelford Medical Practice, Cambridge, UK

Introduction As economic pressures increase, many primary care providers are restricting referrals to hospital. Evidence based guidance for such restrictions are difficult to find and there is a risk that patients with significant disease may have their diagnoses delayed or missed. We have worked with colleagues in primary care to produce a single page guidance sheet for doctors seeing children with common (and occasionally important) symptoms.

Methods We started with a common problem - the coughing child. Most children can be managed in the community, but some have important underlying diseases that need more specialised investigation and treatment. We developed three tools

1. an evidenced algorithm for referral, clarifying which features of the history and examination indicated significant or worrying disease

2. recently published reviews that allowed professionals to read more on the subject,

3. good patient information that set out the principles of our advice.

These were reviewed and discussed at a joint meeting with primary care doctors and then the amended guidelines were disseminated.

Results The resultant guideline (one page with links to articles and information sheets) has been popular with primary care, and is available through the hospital website. It has been used in training. Involvement of primary care doctors has improved its credibility, and early analysis of referral patterns suggests that practices using the guidelines have a lower referral rate to secondary care than the local average, suggesting it modifies referral behaviour. We hope to employ the methodology in a wide range of common paediatric conditions.

\section{MATERNAL ACCESS TO COMMUNICATION IN HEALTH AND HEALTH CARE AND CHILDHOOD IMMUNISATION IN SELECTED PERI-URBAN SETTLEMIENTS IN KUMASI METROPOLIS, GHANA}

doi:10.1136/archdischild-2012-302724.1747

D Buor. Valley View University, Accra, Ghana

The paper examines the effects of mass media health communication, specifically, maternal access and use of media communication in health and health care on childhood immunisation at the periurban settlements in the Kumasi metropolis. A sample of 240 mothers, drawn using random (chance selection) was used for the survey. The main research instrument was formal interview (face-to-face). Charts were used to depict the results whilst chi square tests, derived from cross-tabulation using the Statistical Package for Social Scientists (SPSS), were used to determine significant differences among the independent variables. Results show that children whose parents regularly access mass media health communication go through all or greater part of immunisation methods. Other factors that influence childhood immunisation are maternal age, schooling, employment, marital status and husband's education. For maternal access of health communication, the significant factors influencing it are maternal education, husband's education and marital status. Recommendations made to improve maternal access of childhood immunisation facilities and health communication include, compulsory education of the girl-child beyond basic education, informal education of illiterate mothers, provision of mass media health communication facilities such as television sets in the communities, enhancement of health communication at the hospitals, the simplification of the language used in health communication, and the regular use of local languages. Further research areas have been proposed.
1748 OPEN ACCESS TO HOSPITAL: A WAY TO IMPROVE CARE OF CHILDREN WITH CHRONIC CONDITIONS

doi:10.1136/archdischild-2012-302724.1748

H Bentur, SP Hulikere. Paediatrics, Warrington and Halton Hospitals NHS Foundation Trust, Warrington, UK

Background and Aims Children with chronic conditions have complex healthcare needs. They need a service which ensures easy access with continuity of care while providing co-ordination and support for self-management. Open access to hospital provides one such service and we reviewed our existing local practice to gain insight and improve our service.

Methods Study was conducted at Warrington General Hospital, which is a busy district general hospital in United Kingdom. This is a retrospective review of patients who have an open access to children's ward. Patient information regarding the lead consultant, diagnosis, management plan and advice available from open access folder were reviewed.

Results Total number of patients with open access to children's ward were $182.95 \%$ had information regarding their diagnosis, $80 \%$ had named lead consultant. Only 35\% had information on advice and management, and $30 \%$ had information regarding patient medication. There was overall poor documentation and poor information on patient details. It was also noted that there was no consistency by the consultants regarding the layout of the open access letter.

Conclusion Patient information and management plan were incomplete in majority. Our data shows the need to identify these children with chronic complex needs who can access hospital easily, have a system to keep up-to date information which will help in provision of better care and service, and share information among different professionals.

Based on these findings, we have made recommendations to improve the local practice and information management.

\section{SAFETY NETTING INFORMATION FOR THE ACUTELY SICK CHILD: SYSTEMATIC LITERATURE ON EFFECTIVENESS}

doi:10.1136/archdischild-2012-302724.1749

${ }^{1} \mathrm{M}$ Lakhanpaul, ${ }^{2} \mathrm{~S}$ Neill, ${ }^{2} \mathrm{C}$ Shang, ${ }^{3} \mathrm{M}$ Thompson, on behalf of the ASK SNIFF Team. ${ }^{1}$ General and Adolescent Paediatric Unit, University College London, Institute of Child Health, London; 'University of Northampton, Northampton; ${ }^{3 P r i m a r y}$ Care Health Sciences, University of Oxford, Oxford, UK

Background and Aims Uncertainty and anxiety surround parents' decisions to seek medical help for a sick child at home because parents are concerned not to miss serious illness whilst also not bothering the doctor unnecessarily. This dilemma leads to parents' desire for more information to support their decision making. Factors influencing the success of information resources need to be identified prior to the development of safety netting interventions for families. The ASK SNIFF team (Acutely Sick Kid Safety Netting Interventions for Families) aimed to review the literature on the effectivenss of existing resources to provide an evidence base for the develoment of safety netting information for parents to be used when determining to seek help for an acutely sick child.

Methods The initial stage of the project involved a systematic review using narrative analysis to identify influences on the effectiveness of information resources for parents caring for an acutely ill child at home.

Results A range of measures for effectiveness have been used in the literature which limits their comparison. Interventions that included information on more than one illness or symptom were found to be more effective. Parents were more likely to act on information provided to them in the community than in the emergency 
department. Co- created information by parents and health professionals were found to be more successful.

Conclusions Safety netting information resources may be more effective if they are designed with parents and their content, mode and place delivery is evidence based.

\section{IMPLEMENTING DEVELOPMENTAL SCREENING RECOMMENDATIONS IN A FAMILY MEDICINE RESIDENCY TRAINING PROGRAM: BARRIERS, SOLUTIONS AND RECOMMENDATIONS FOR PRACTICE}

doi:10.1136/archdischild-2012-302724.1750

'GA Devito, $2 \mathrm{~L}$ Manning, ${ }^{2} \mathrm{P}$ McLean, ${ }^{3} \mathrm{~A}$ Valeras. 'Department of Pediatrics Concord Hospital, New Hampshire Dartmouth Family Medicine Residency, Dartmouth Medical School; '2Family Health Center, NH Dartmouth Family Medicine Residency Program; ${ }^{3}$ Family Health Center, NH Dartmouth Family Medicine Residency, Dartmouth Medical School, Concord, NH, USA

Background and Aims The American Academy of Pediatrics recommends that all children, as part of well child care, have validated developmental screening in the medical home. Testing increases the early identification of children with developmental delay and improves outcome. However, USA data show that many primary care practices do not provide this screening. Our goal was to understand these barriers in our own practice, to overcome them, and to achieve $100 \%$ developmental screening rates.

Methods Our OI workgroup used electronic surveys and focus group discussion to solicit parent expectations, to understand provider barriers, and to explore work flow issues. Using this information to drive PDSA cycles and LEAN initiatives in our practice, we instituted educational training, workflow adjustments, screening tool modification, computerized tracking, and the redesign of select well child visits to focus on development screening.

Results The use of a validated screening tool by non-pediatric providers in our practice was $0 \%$ at the initiation of intervention. The major barriers to screening were lack of provider time, lack of comfort in developmental/behavioral pediatrics, lack of familiarity with a screening tool, and perceived lack of need for formalized testing. Following implementation of changes, including the modification of specific well visits to focus on developmental screening, $100 \%$ of eligible children had validated developmental screening performed over the last two months.

Conclusion Educational intervention, work flow modification, and the refocus of select well visits can significantly improve developmental screening rates in practice.

\section{CHILDPROOFING CANNOT BE SAFELY ASSUMED AFTER ACCIDENTAL POISON INGESTION RELATED HOSPITAL VISITS}

doi:10.1136/archdischild-2012-302724.1751

S Bharwani. Pediatrics, Faculty of Medicine and Health Sciences, UAE University, Al Ain, United Arab Emirates

Background/aims: Data is scant on the follow up of the patients after accidental poison ingestions. We wanted to determine if the parents of children with recent accidental poison ingestions took any steps for childproofing their homes after discharge from the hospital.

Method We conducted telephone interviews with the parents of children who visited ER (emergency center) of a tertiary center for accidental poison ingestions in the preceding 3-13 months. The parents were asked if they lock medicines in the cabinets, keep cleaning materials out of the reach of young children, educate their domestic helpers or do nothing to prevent such accidents. The parents had the option of selecting more than one strategy.
Results 100 parents were contacted and 71 decided to participate. 62 out of 71 households had a domestic helper but only 22/71 parents $(31 \%)$ said they would educate their domestic helpers. 39 parents $(55 \%)$ said they did or would lock the medicines in the cabinets and 35 out of $71(49 \%)$ said they would or did keep the cleaning materials out of reach of young children. Only 16 parents out of $71(22.5 \%)$ said they would do or did both- lock the medicines in the cabinet and keep the cleaning supplies out of reach of their young children.

Conclusion The domestic and behavior changes are not a foregone conclusion after accidental poison ingestions related ER visits. Follow up home visitation by a public health nurse for personalized tips in childproofing may have a positive behavioral outcome.

\section{A NATIONAL PROPOSAL IN COMMUNITY RISK PREVENTION: TRANSITION TO ADULT HEALTH CARE FOR ADOLESCENTS WITH CHRONIC DISEASES}

doi:10.1136/archdischild-2012-302724.1752

0 Urroz. National Cancer Institute Childrens Hospital, San Jose, Costa Rica

Childrens with complex chronic diseases now survive past the age of adolescence and into adult life, however health services have not always recognized the need to prepare these patients for the move into their specific adult services. This issue has resulted in these adolescents having to find a way to obtain the necessary health care services from adult healthcare providers However, without proper preparation on both ends of healthcare, this transition is very difficult. For all adolescents, the transition from childhood to adulthood involves combining the patient's individual identity, need to achieve independence, establishment of adult relationships and obtainment of a meaningful occupation. This transition is made more difficult because of the patient's continuous concerns about whether their social and health care needs will be met. These are the more serious reasons why many adolescents with chronic diseases are greatly hindered during the transition process. The effects of these issues are the debilitation and deterioration of the patient's health, both physical and psychological, mostly due to the fact that the patient is not prepared properly for the transition to adulthood with regards to their healthcare. The goal in this process is to make sure that the adolescent with chronic diseases, as well as their respective family members, are trained, helping to facilitate the ability to accept and understand that the transition process is like any other stage in life, with the inclusion of implication and new responsibilities specific to the patient that will need to be incorporated quickly and effectively.

\section{THE PERCEPTION OF THE TERM CEREBRAL PALSY (CP) IN SAUDI ARABIA}

doi:10.1136/archdischild-2012-302724.1753

S Madi, A Mandy, T Pountney. University of Brighton, Eastbourne, UK

Background and purposes: Cerebral palsy (CP) is one of the most common childhood disabilities and makes heavy demands on health, educational, and social services as well as on families and children. In Saudi Arabia the term CP is recognized by most health professionals as a physically disabling condition, however this is not the case for the general public, nor more importantly for mothers of children with CP. Misinterpreting the term of CP by Arabic language, clearly exists. This research aimed to explore the perception of the term CP with mothers of children with CP.

Methods Critical ethnography was adopted as the methodological approach; data were collected through focus groups, individual interviews, field note and participant observation. 